\title{
Downregulation of transient receptor potential cation channel, subfamily $C$, member 1 contributes to drug resistance and high histological grade in ovarian cancer
}

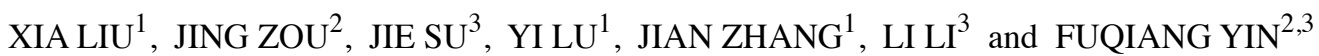 \\ ${ }^{1}$ Center for Translational Medicine, and ${ }^{2}$ Medical Scientific Research Centre, Guangxi Medical University, \\ Nanning, Guangxi 530021; ${ }^{3}$ Key Laboratory of High-Incidence-Tumor Prevention and Treatment \\ (Guangxi Medical University), Ministry of Education, Nanning, Guangxi 530021, P.R. China
}

Received September 21, 2015; Accepted October 15, 2015

DOI: 10.3892/ijo.2015.3254

\begin{abstract}
Transient receptor potential cation channel, subfamily $\mathrm{C}$, member 1 (TRPC1) participates in many physiological functions but has also been implicated in cancer development. However, little is known about the role of TRPC1 in ovarian cancer (OC), including the drug resistance of these tumors. In the present study, a significant and consistent downregulation of TRPC1 in drug-resistant OC tissues/cells was determined using real-time quantitative polymerase chain reaction assays and the microarrays deposited in Oncomine and Gene Expression Omnibus (GEO) Profiles. Protein/geneprotein/gene and protein-chemical interactions indicated that TRPC1 interacts with 14 proteins/genes and 6 chemicals, all of which are involved in the regulation of drug resistance in OC. Biological process annotation of TRPC1, OC, and drug resistance indicated a role for TRPC1 in drug-resistance-related functions in $\mathrm{OC}$, mainly via the cell cycle, gene expression and cell growth and cell death. Analysis of mRNA-microRNA interactions showed that 8 out of 11 major pathways enriched from 38 predominant microRNAs targeting TRPC1 were involved in the regulation of drug resistance in $\mathrm{OC}$, and 8 out of these top 10 microRNAs were implicated in the drug resistance in ovarian and other cancers. In a clinical analysis using data obtained from The Cancer Genome Atlas project (TCGA) cohort on 341 OC patients, TRPC1 expression was found to differ significantly between grade 2 and grade 3 tumors, with low-level expression correlating with higher tumor grade. This is the first report to show a potential association between the downregulation of TRPC1 and both drug resistance and high
\end{abstract}

Correspondence to: Dr Fuqiang Yin, Medical Scientific Research Centre, Guangxi Medical University, 22 Shuangyong Road, Nanning, Guangxi 530021, P.R. China

E-mail: yinfq@mail2.sysu.edu.cn

Key words: Transient receptor potential cation channel, subfamily C, member 1 , ovarian cancer, drug resistance, histological grade, bioinformatics histological tumor grade in OC. Our results provide the basis for further investigations of the drug-resistance-related functions of TRPC1 in OC and other forms of cancer.

\section{Introduction}

The treatment of ovarian cancer (OC) has improved over the past 30 years, following the introduction of platinum- and paclitaxel-based chemotherapy. However, most patients with $\mathrm{OC}$ will suffer disease relapse despite having achieved a complete clinical response. In many of these patients, the disease is incurable mainly owing to the development of drug resistance (1). Treatment failure and death from $\mathrm{OC}$ have been attributed to drug resistance in $>90 \%$ of patients with metastatic disease. Thus, a better understanding of the mechanisms of drug resistance in OC will lead to improved treatment strategies and perhaps, better survival (2).

Drug resistance in $\mathrm{OC}$ is the product of a myriad of contributing factors (3). These include microRNA and long non-coding RNA regulation, increased DNA damage tolerance/repair, increased or altered drug targets, growth factor receptor deregulation, increased anti-apoptotic regulator activity and metabolic disturbances (4-7). Underlying or accompanying these factors is the dysregulation of critical genes (8). Richardson and Kaye (9) described several gene families that may contribute to the evolution of drug resistance in OC, via pathways involving DNA damage, apoptosis and cell-survival signaling. In previous studies, we identified 15 tumor suppressor genes (TSGs) and 25 oncogenes that contribute to drug resistance in OC through DNA damage, apoptosis, cell cycle interactions, DNA binding and the AKT and BAX-mediated signaling pathways $(8,10)$. Thus, the identification of drug-resistance-related genes and investigation of their contribution to drug resistance may offer solutions to the successful treatment of patients with OC.

The cellular entry of extracellular $\mathrm{Ca}^{2+}$ and the subsequent elevation of the intracellular $\mathrm{Ca}^{2+}$ concentration are key events in cell cycle progression (11). Indeed, $\mathrm{Ca}^{2+}$ mediated signaling is associated with many features of cancer, including the development of drug resistance $(12,13)$. Cellular $\mathrm{Ca}^{2+}$ entry is mediated by transient receptor poten- 
tial (TRP) proteins. These are non-selective cation channels that are permeable to $\mathrm{Ca}^{2+}(14)$ and fulfill diverse roles as versatile cellular sensors and effectors (15). TRPC1 was the first cloned mammalian TRP protein. It is found in a wide variety of tissues, where it contributes to physiological functions such as cell proliferation, differentiation, cell migration, membrane permeability, fluid secretion, smooth and skeletal muscle function, wound healing and protection against cell death (16). Moreover, TRPC1 is also involved in the regulation of cancer development and was shown to play an important role in apoptosis in hepatocellular carcinoma (17), the metastasis of nasopharyngeal carcinoma (18), and in promoting cell proliferation in non-small cell lung carcinoma cell lines (19). In OC, TRPC1 was shown to be essential in promoting cell proliferation and tumorigenesis (20). However, the exact role of TRPC1 in cancer is poorly understood and whether it contributes to drug resistance in OC has never been determined.

In the present study, we provide evidence of a marked decrease in TRPC1 mRNA levels in human OC vs. normal specimens/cells and of their downregulation in drug-resistant OC vs. sensitive cells. Comprehensive bioinformatics analyses suggested the interaction of TRPC1 with numerous proteins/ genes, chemicals, biological processes and microRNAs, all of which are involved in the regulation of OC drug resistance. In addition, lower TRPC1 expression was shown to correlate with the high histological grade of tumors in OC patients.

\section{Materials and methods}

Cell culture. The human epithelial OC cell lines SKOV3 and A2780 were maintained in our laboratory and propagated in vitro by serial passage in RPMI-1640 medium supplemented with $10 \%$ fetal bovine serum (FBS). The cisplatin-resistant cell line SKOV3-DDP and the carboplatin-resistant cell line A2780-CBP were established by sequential exposure of cells to increasing concentrations of cisplatin and carboplatin, respectively. The resistance index of the SKOV3-DDP and A2780-CBP OC cells is 2.4 and 2.0, respectively.

Real-time quantitative polymerase chain reaction analysis. Total RNA was isolated from the cell lines SKOV3, SKOV3-DDP, A2780 and A2780-CBP, using TRIzol reagent (Life Technologies, Grand Island, NY, USA). The quantity and quality of the RNA were measured using a Thermo Scientific NanoDrop 2000 spectrophotometer (Thermo Fisher Scientific, Wilmington, DE, USA). cDNA was synthesized from $2 \mu \mathrm{g}$ of RNA using the SuperScript III First-Strand Synthesis system (Life Technologies). TRPC1 mRNA levels were measured using real-time quantitative polymerase chain reaction (RT-qPCR) and the Power SYBR-Green PCR Master Mix (Applied Biosystems, Life Technologies, Waltham, MA, USA). Data were collected with the Applied Biosystems 7300 RT-PCR system in accordance with the manufacturer's instructions. The RT-qPCR gene-specific primers for TRPC1 were: (forward primer) 5'-ACGTCTAGTGACGAGCCTCT-3' and (reverse primer) 5'-CCCGACATCTGTCCAAACCA-3'. For GAPDH, used as the control, the forward primer was 5'-GAAGGTGAAGGTCGGAGT-3' and the reverse primer 5'-GAAGATGGTGATGGGATTT-3'.
Gene expression profiles. TRPC1 mRNA expression data were retrieved from Oncomine (https://www.oncomine.org/ resource/login.html) (21) and GEO Profiles (http://www. ncbi.nlm.nih.gov/geoprofiles/) (22). Welsh Ovarian Statistics, Bonome Ovarian Statistics and Yoshihara Ovarian Statistics data are deposited in Oncomine. The level of TRPC1 mRNA expression between OC and normal controls in the Oncomine microarrays are presented as fold changes; significant differences are indicated based on their P-value. Microarrays GDS3592, GDS3754 and GDS1381 are deposited in GEO Profiles. In this database, in the case of two probe sets targeting one gene, only the probe set with significant statistical variability was retained. In the case of more than three probe sets targeting one gene, the set exhibiting the most divergent expression was excluded and the set with significant statistical variability was retained. The expression data of TRPC1 and microRNAs, and clinical data of 489 OC patients registered in The Cancer Genome Atlas (TCGA) cohort was retrieved from the cBioPortal for Cancer Genomics (http://cbioportal. org) $(23,24)$.

Bioinformatics analyses. Protein/gene interactions were determined using the GeneMANIA online tool (http://www. genemania.org/) (25). Protein/small-molecule/chemical interactions were analyzed using STITCH (http://stitch.embl. de/) (26,27). Biological processes were annotated using the Coremine Medical tool (http://www.coremine.com/medical/) (28). Interactions between microRNA and mRNA were predicted using miRWalk (http://www.umm.uni-heidelberg.de/ apps/zmf/mirwalk/) (29), for which eight prediction programs (DIANAmT, miRanda, miRDB, miRWalk, RNAhybrid, PICTAR5, RNA22 and TargetScan) were selected; the same microRNA predicted by at least six of them was selected for further analysis. Pathway enrichment analysis of microRNAs was performed using the DIANA-mirPath web server (http:// diana.cslab.ece.ntua.gr/pathways/) (30).

Data analysis. The data were analyzed using the SPSS 20.0 software. The mRNA expression level of a particular gene is shown as the mean $\pm \mathrm{SD}$. The homogeneity of the variance was analyzed using the t-test. The probability of survival and significance were calculated using the Kaplan-Meier method and a log-rank test. The correlation between microRNAs and the gene was analyzed using bivariate correlations. The correlation between gene expression and the clinicopathological characteristics was evaluated by Pearson's $\chi^{2}$ test (2-sided). Expression values of a gene were dichotomized into high and low expression using the median as a cut-off in a Kaplan-Meier analysis, in accordance with previous studies $(31,32)$. A P-value $<0.05$ was considered to indicate statistical significance.

\section{Results}

TRPC1 is significantly downregulated in OC and drug-resistant OC tumor specimens/cells. TRPC1 mRNA expression was significantly and consistently downregulated in OC tissues and cells compared with the expressions in normal controls, as determined using data retrieved from microarrays deposited in Oncomine and GEO Profiles. As indicated in Fig. 1A, TRPC1 mRNA expression was downregulated by 3.955-, 3.681- and 


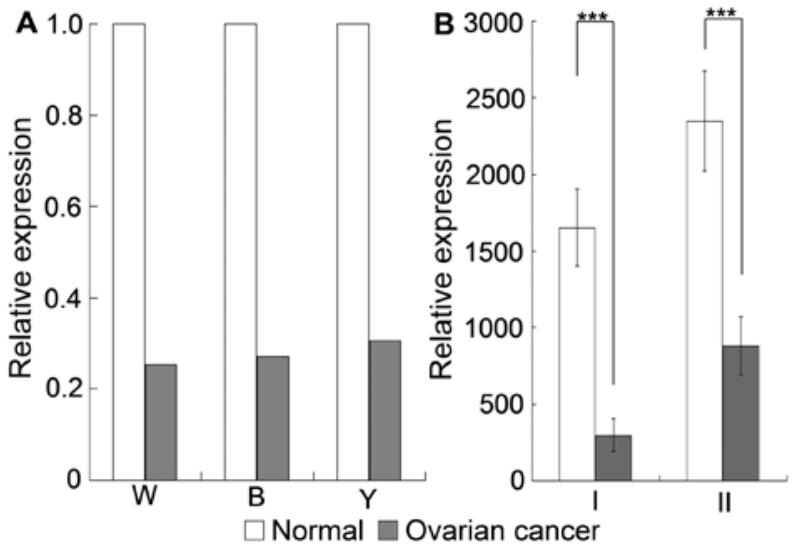

Figure 1. TRPC1 mRNA expression in ovarian cancer (OC) tissues and cells compared with their normal controls. (A) Expression was determined using microarray data retrieved from Oncomine. W, Welsh ovarian microarray. TRPC1 expression is downregulated 3.955 -fold $(\mathrm{P}=2.98 \mathrm{E}-10)$ in the 28 ovarian serous surface papillary carcinomas compared to the four tissues from normal ovaries. (B) Bonome ovarian microarray. TRPC1 expression is downregulated 3.681 -fold $(\mathrm{P}=6.84 \mathrm{E}-9)$ in the 185 ovarian carcinomas compared with the 10 normal ovarian surface epithelium samples. Y, Yoshihara ovarian microarray. TRPC1 expression is downregulated 3.260fold $(\mathrm{P}=2.08 \mathrm{E}-9)$ in the 38 ovarian serous adenocarcinomas compared with the 10 peritoneal tissues. (B) TRPC1 mRNA expression in normal ovarian surface epithelia and in OC epithelial cells as determined using microarray data (GDS3592) retrieved from GEO Profiles. Normal ovarian surface epithelial cells were collected from ovaries at the time of surgery using a Cytobrush Plus; tumor tissues were surgically removed and collected for cell isolation. The normalized data were deposited in GEO Profiles (33). Twelve biological replicates were obtained for the normal cells and tumor cells. I, GDS3592/205803_s_at/TRPC1; II, GDS3592/205802_at/TRPC1. ${ }^{* * *} \mathrm{P}<0.001$.

3.260-fold in OC specimens according to the Oncomine Welsh ovarian microarray, covering 28 ovarian serous surface papillary carcinomas and four normal ovarian tissues; the Bonome ovarian microarray, covering 185 ovarian carcinomas and 10 ovarian surface epithelia; and the Yoshihara ovarian array, covering 38 ovarian serous adenocarcinomas and 10 peritoneal tissues. Consistent with the expression in OC specimens, TRPC1 expression was downregulated by at least 3-fold in OC cells compared with the expression in normal ovarian surface epithelial cells, in accordance with GEO Profiles analyses (Fig. 1B). There was also a significant downregulation of TRPC1 mRNA expression in A2780 epithelial OC cells with acquired platinum resistance and in carboplatin-resistant OC cells compared with expression in the sensitive counterparts of both (Fig. 2A and B). This result was confirmed by the RT-qPCR analysis, in which TRPC1 mRNA expression was significantly downregulated in both cisplatin-resistant SKOV3 cells and carboplatin-resistant A2780 cells compared with expression in the corresponding sensitive cells (Fig. 2C). These results suggested that the stable and significant downregulation of TRPC1 in specimens/cells from OC and drug-resistant OC plays a critical role in the development drug resistance of these tumors.

Comprehensive analyses indicating potential associations of TRPCl with drug resistance in $O C$

Functional prediction and analysis based on protein/geneprotein/gene interactions. The protein/gene interactions of
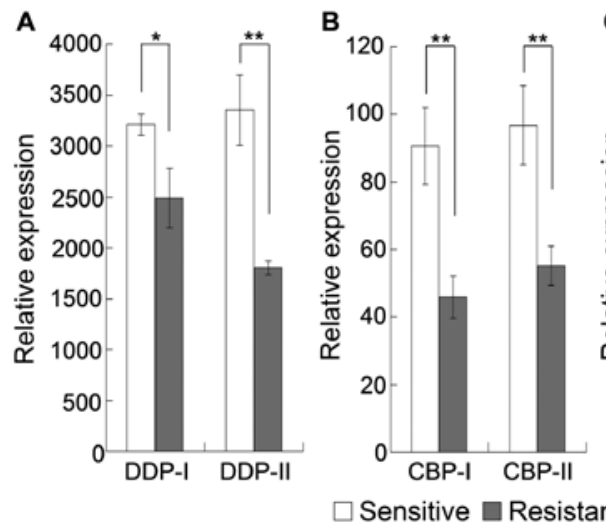

C

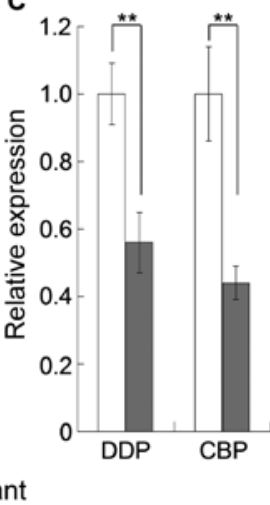

Figure 2. TRPC1 mRNA expression in drug-sensitive and drug-resistant OC cells as determined using microarray data retrieved from GEO Profiles and RT-qPCR analysis. (A) TRPC1 mRNA expression in drug-sensitive A2780 epithelial OC cells and cells with acquired platinum resistance, demonstrated using microarray data (GDS3754). Five replicates were performed for each cell line using Human U133 plus 2.0 GeneChips (Affymetrix, Santa Clara, CA, USA). The normalized data were deposited in GEO Profiles (34). DDP-I, GDS3754/205802_at/TRPC1; DDP-II, GDS3754/205803_s_at/TRPC1.

(B) TRPC1 mRNA expression in carboplatin-sensitive and resistant ovarian cancer cells as shown using microarray data (GDS1381). Primary cultures of cells derived from ovarian carcinomas of individual patients $(n=6)$ were characterized using the ChemoFx assay and classified as either carboplatin sensitive $(n=3)$ or resistant $(n=3)$. Three representative cultures of cells from each individual tumor were subjected to Affymetrix GeneChip analysis $(\mathrm{n}=18)$. The normalized data were deposited in GEO Profiles (35). CBP-I, GDS1381/39123_s_at/TRPC1; CBP-II, GDS1381/39124_r_at/TRPC1. (C) TRPC1 mRNA expression in cisplatin- and carboplatin-resistant OC cells measured by RT-qPCR. Four replicates were performed for the drugresistant cells and for the controls. DDP, cisplatin-sensitive and -resistant SKOV3 OC cells; CBP, carboplatin-sensitive and -resistant A2780 OC cells. ${ }^{*} \mathrm{P}<0.05 ;{ }^{* *} \mathrm{P}<0.01$.

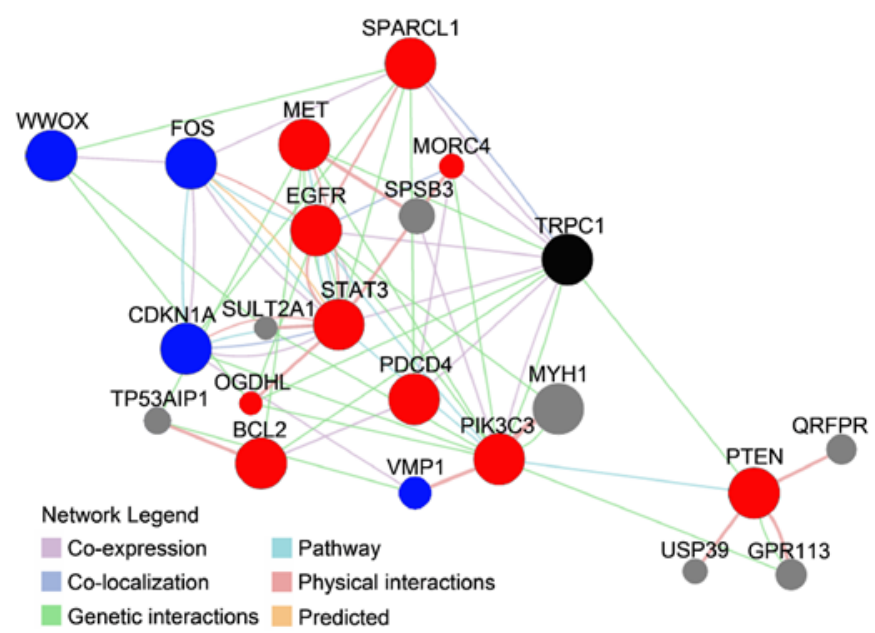

Figure 3. Protein/gene-protein/gene interaction network of TRPC1 generated using the GeneMANIA tool. The query in red includes the proteins/genes that interact directly with TRPC1, and the query in blue includes the drug resistant-related proteins/genes that interact indirectly with TRPC1. The types of interactions between proteins/genes are indicated in the network legend of the figure.

TRPC1 with other proteins/genes were analyzed using the GeneMANIA tool. As shown in Fig. 3, TRPC1 interacted directly with 10 genes/proteins: MORC family CW-type zinc finger 4 (MORC4), epidermal growth factor receptor (EGFR), signal transducer and activator of transcription 3 (STAT3), 


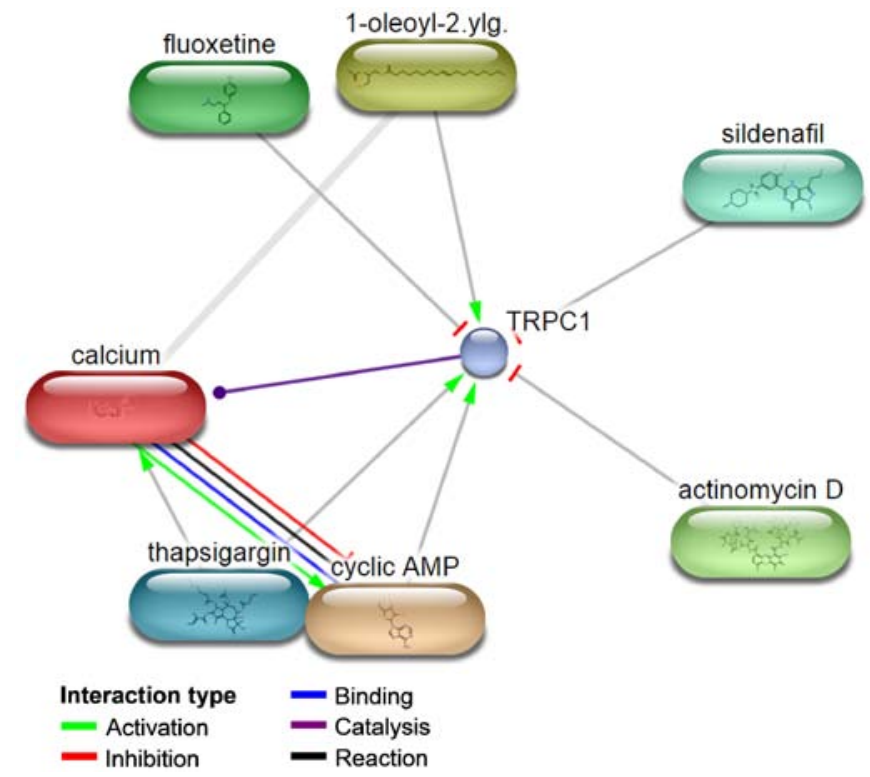

Figure 4. Protein and small molecule/chemical interaction analysis of TRPC1 using STITCH (version 4.0). The types of interaction between TRPC1 and chemicals are shown. 1-oleoyl-2.ylg., 1-oleoyl-2-acetylglycerol.

programmed cell death 4 (PDCD4), MET proto-oncogene, receptor tyrosine kinase (MET), oxoglutarate dehydrogenaselike (OGDHL), B-cell CLL/lymphoma 2 (BCL2), phosphatase and tensin homolog (PTEN), SPARC-like 1 (hevin) (SPARCL1) and phosphatidylinositol 3-kinase, catalytic subunit type 3 (PIK3C3). Except for MORC4, all of these genes/proteins have been implicated in the regulation of drug resistance in OC. For example, PTEN is a TSG involved in the regulation of drug resistance via the PI3K/AKT pathway and the p53-mediated apoptotic cascade. A reduction in PTEN expression confers resistance to cisplatin in OVCAR-3 cells through the activation of PI3K/Akt (36), and the overexpression of PTEN reverses chemoresistance to cisplatin in human OC cells by inactivating the PI3K/AKT cell survival pathway (37). However, in another study, the overexpression of PTEN upregulated p53 and increased the sensitivity of chemoresistant cells to cisplatin-induced apoptosis without detectable changes in the levels of phosphorylated Akt, suggesting that PTEN regulates drug resistance through a p53-mediated apoptotic cascade independent of the PI3K/Akt pathway (38). In a recent study, the overexpression of PTEN improved the cisplatin-resistance of human OC cells by upregulating KRT10 expression. Thus, the exogenously induced overexpression of KRT10 may be a therapeutic strategy for overcoming multi-drug resistance in OC (39). BCL2, STAT3, EGFR and MET are drug-resistancerelated oncogenes (10), and PDCD4 (40) and SPARCL1 (41) are drug-resistance-related TSGs. All of these genes are expressed in OC. OGDHL is thought to modify AKT-dependent signaling and NFKB1 function (42), both of which play crucial roles in the regulation of drug resistance in OC (10).

In addition to proteins/genes that directly interact with TRPC1, there were others in the network whose interaction with TRPC1 was indirect (Fig. 3). Among those, CDKN1A (43,44), FOS $(45,46)$, WWOX (47) and VMP1 (48) are associated with drug resistance in OC. Collectively, among the 21 proteins/genes found to interact with TRPC1, 14 were

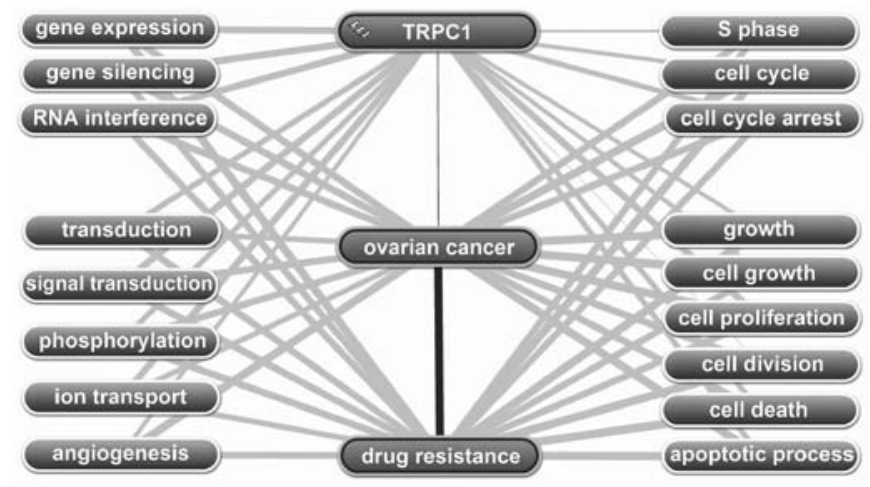

Figure 5. Biological process annotation of TRPC1, ovarian cancer (OC) and drug resistance $(\mathrm{P}<0.01)$. The input terms were TRPC1, OC and drug resistance.

associated with drug resistance in OC. These results strongly suggest an association between TRPC1 and drug resistance in OC.

Prediction and analysis of function based on protein and small-molecule/chemical interactions. The protein and small molecule/chemical interaction was assessed in an attempt to further explain the drug-resistance-related functions of TRPC1 in OC. As shown in Fig. 4, TRPC1 interacts with 1-oleoyl-2-acetylglycerol, cyclic AMP, thapsigargin, calcium, actinomycin D, fluoxetine and sildenafil. The first three compounds activate TRPC1 and the last three inhibit TRPC1. Except for sildenafil, all of these compounds are associated with drug resistance or are otherwise involved in chemotherapy in ovarian and other cancers. For example, 1-oleoyl-2-acetylglycerol enhances the phosphorylation of P-glycoprotein (49), which contributes to the development of drug resistance in OC by regulating several pathways, such as nuclear factor of kappa light polypeptide gene enhancer in B-cells 1 (NFKB1), mitogen-activated protein kinase (MAPK) and phosphatidylinositol-4,5-bisphosphate 3-kinase, and catalytic subunit alpha (PI3K) signaling (50). Cyclic AMP reduces the induction of AP-1 binding, required for the activation of interleukin 8 (IL8) by paclitaxel (51). The presence of IL8 in paclitaxel-treated OC cells contributes to the development of paclitaxel resistance (52). In a recent study, thapsigargin exhibited pharmacological activity against OC stem cells (53). Fluoxetine is an antidepressant that greatly enhances the cytotoxicity of cisplatin in HCT116 wild-type (wt), HCT116 (p53-/-), HT-29, SKOV3 and A2780 cells (54) and induces apoptosis through the reactive oxygen species-dependent activation of NFKB1, frequently implicated in the regulation of drug resistance (55). Actinomycin D is an antitumor agent used in the chemotherapy of OC (56). Thus, among the identified small molecules, 1-oleoyl-2-acetylglycerol, cyclic AMP, thapsigargin, actinomycin D and fluoxetine are drugresistance-related or are involved in the chemotherapy of OC. Their interaction with TRPC1 implicates this protein in the drug resistance of these tumors. There is also evidence for a relationship between calcium and drug resistance in OC. A calcium channel blocker verapamil was reported to reverse adriamycin resistance through inhibition of adriamycin efflux in OC resistant cells (57). Similarly, doxorubicin resistance is 
Table I. Top 11 enriched pathways modulated by microRNAs targeting TRPC1 and their associations with drug resistance in ovarian cancer.

Regulation of

drug resistance in

MicroRNAs KEGG pathway $\quad$ P-value ovarian cancer (Refs.)

hsa-miR-

135b, 135a, 603, 186, 497, 548b-3p,

188-5p, 612, 26a, 103, 10b, 570,

590-3p, 195, 548c-5p, 324-3p, 410,

$107,573,142-5 \mathrm{p}, 548 \mathrm{c}-3 \mathrm{p}, 15 \mathrm{a}, 338-5 \mathrm{p}$,

16, 577, 548a-5p, 543, 548d-3p, 424, 559,

$605,340,943,495,15 \mathrm{~b}, 607,101,641$

Pathways in cancer
PI3K-Akt signaling pathway
MAPK signaling pathway
HTLV-I infection
Regulation of actin cytoskeleton
Endocytosis
Focal adhesion
Transcriptional misregulation in cancer
Wnt signaling pathway
Protein processing in endoplasmic reticulum
Hepatitis B

Pathways in cancer

MAPK signaling pathway

HTLV-I infection

Endocytosis

Focal adhesion

Wnt signaling pathway

Hepatitis B

$\begin{array}{ll}4.12 \mathrm{E}-29 & \text { Cancer pathway } \\ 3.57 \mathrm{E}-27 & \text { Yes }(36,70) \\ 1.20 \mathrm{E}-21 & \text { Yes }(71,72) \\ 1.83 \mathrm{E}-20 & - \\ 2.20 \mathrm{E}-18 & \text { Yes }(73) \\ 3.88 \mathrm{E}-18 & \text { Yes }(74) \\ 1.29 \mathrm{E}-17 & \text { Yes }(75) \\ 2.08 \mathrm{E}-17 & \text { Cancer pathway } \\ 3.72 \mathrm{E}-15 & \text { Yes }(76) \\ 3.72 \mathrm{E}-15 & - \\ 3.95 \mathrm{E}-15 & -\end{array}$

The microRNAs predicted by at least six of the eight prediction tools (DIANAmT, miRanda, miRDB, miRWalk, RNAhybrid, PICTAR5, RNA22 and TargetScan) were submitted to pathway enrichment using DIANA miRPath (30).

reversed by verapamil (58). Since TRPC1 is an activator of calcium, these results also suggest its involvement in drug resistance.

Prediction and analysis of function based on the annotation of biological processes. The Gene Ontology (GO) consortium (59) provides a valuable source of structured knowledge of protein function in terms of molecular function, biological processes and cellular components. A gene may be involved in one or more biological processes (60), and the involvement of a gene in a given biological process can be used to predict the biological role and function of that gene (61). The Coremine Medical online database can be publicly accessed by anyone seeking information on health, medicine and biology $(28,62)$. It can be used to analyze the associations between genes, biological processes and drug resistance. As shown in Fig. 5, 17 biological processes significantly associated with TRPC1, $\mathrm{OC}$ and drug resistance were annotated $(\mathrm{P}<0.01)$. Given the close relationships of TRPC1 with these processes, and their close relationships with OC and drug resistance, TRPC1 is likely to be involved in the regulation of drug resistance in $\mathrm{OC}$. Six of those processes are cell-growth- and cell death-related, three are cell cycle-related and 3 are gene expression-related (Fig. 5). Thus, the drug-resistance-related functions of TRPC1 in $\mathrm{OC}$ are likely to reflect its involvement in the cell cycle, gene expression, and in particular, cell growth and cell death.

Functional prediction based on the functionality of microRNAs that target the TRPC1 gene. MicroRNA-mediated post-transcriptional gene regulation is an important regulator of many cellular processes, both physiological and pathological $(63,64)$. The target genes of microRNAs are a focus of interest based on their diagnostic, prognostic and therapeutic relevance (65), and the function of a gene can be predicted based on functionality of the microRNAs that target it (41). To identify microRNAs that target TRPC1, a microRNA-mRNA interaction analysis was performed with miRWalk, which resulted in the identification of 481 microRNAs predicted to transcriptionally target TRPC1 and suggested the regulation of TRPC1 by microRNA. Those microRNAs predicted by at least 6 of the 8 prediction tools were submitted to pathway enrichment using DIANA miRPath (30). Pathways involved in the regulation of cancer development and progression are also likely to be involved in the regulation of drug resistance in ovarian and other cancers. As shown in Table I, 11 pathways were enriched from the top 38 microRNAs that potentially target TRPC1. Among the 11 pathways, at least 8 are involved in the regulation of drug resistance in $\mathrm{OC}$, including the PI3K-Akt, MAPK and Wnt pathways. PI3K/AKT signaling is a major cell survival pathway (66). It is involved in the onset of drug resistance in OC (34) by promoting cell survival and blocking apoptosis (36).

To confirm the potential regulation roles of the microRNAs on TRPC1, the correlation between top 10 microRNAs (Table II) and TRPC1 was performed, on the basis of their expressions in ovarian cancer tissues. The expression data of the microRNAs and TRPC1 were retrieved from TCGA cohort covering 489 cases of ovarian cancer tissues. Among the 8 microRNAs (the expression data of miR-603 and miR-103 are not available), 5 of them including miR-135b, miR-186, miR-26a, miR-497 and miR-548b-3p are significantly correlated with TRPC1, in particular the former 2, their high expression is significantly correlated with the low expression of TRPC1 (Fig. 6).

Eight of the top 10 microRNAs that targeted TRPC1 are associated with drug resistance in ovarian and other cancers (Table II). For example, miR-135a is a tumor suppressor in epithelial OC and regulates HOXA10 expression (67), which correlates with platinum resistance in OC (68). Upregulation of miR-497 in Taxol-resistant OC is closely associated with 
Table II. Top 10 microRNAs targeting TRPC1, as predicted by microRNA-mRNA interactions and their drug-resistance-related functions in cancer.

\begin{tabular}{|c|c|c|c|c|c|c|c|c|c|c|}
\hline \multirow[b]{2}{*}{ Gene } & \multirow{2}{*}{$\begin{array}{l}\text { microRNA } \\
\text { (hsa-) }\end{array}$} & \multicolumn{8}{|c|}{ Eight microRNA-mRNA prediction tools } & \multirow{2}{*}{$\begin{array}{l}\text { Drug-resistance-related functions } \\
\text { of the microRNAs in cancers (Refs.) }\end{array}$} \\
\hline & & A & B & $\mathrm{C}$ & $\mathrm{D}$ & $\mathrm{E}$ & $\mathrm{F}$ & G & $\mathrm{H}$ & \\
\hline \multirow[t]{10}{*}{ TRPC1 } & $\mathrm{miR}-135 \mathrm{~b}$ & 1 & 1 & 1 & 1 & 1 & 1 & 1 & 1 & Drug resistance-related (77) \\
\hline & miR-135a & 1 & 1 & 1 & 1 & 1 & 1 & 1 & 1 & Drug resistance-related $(67,68)$ \\
\hline & miR-603 & 1 & 1 & 1 & 1 & 0 & 1 & 1 & 1 & Cancer risk $(78,79)$ \\
\hline & $\operatorname{miR}-186$ & 1 & 1 & 0 & 1 & 1 & 1 & 1 & 1 & Drug resistance-related (80) \\
\hline & $\operatorname{miR}-497$ & 1 & 1 & 1 & 1 & 0 & 1 & 0 & 1 & Drug resistance related $(69,81)$ \\
\hline & miR-548b-3p & 1 & 1 & 1 & 1 & 0 & 1 & 0 & 1 & - \\
\hline & miR-188-5p & 1 & 1 & 1 & 1 & 0 & 1 & 0 & 1 & Drug resistance-related (82) \\
\hline & miR-612 & 1 & 1 & 1 & 1 & 0 & 1 & 0 & 1 & Drug resistance-related (83) \\
\hline & miR-26a & 1 & 1 & 0 & 1 & 1 & 1 & 0 & 1 & Drug resistance-related $(84,85)$ \\
\hline & miR-103 & 1 & 1 & 0 & 1 & 0 & 1 & 1 & 1 & Drug resistance-related (86-88) \\
\hline
\end{tabular}

A, DIANAmT; B, miRanda; C, miRDB; D, miRWalk; E, RNAhybrid; F, PICTAR5; G, RNA22; H, TargetScan.
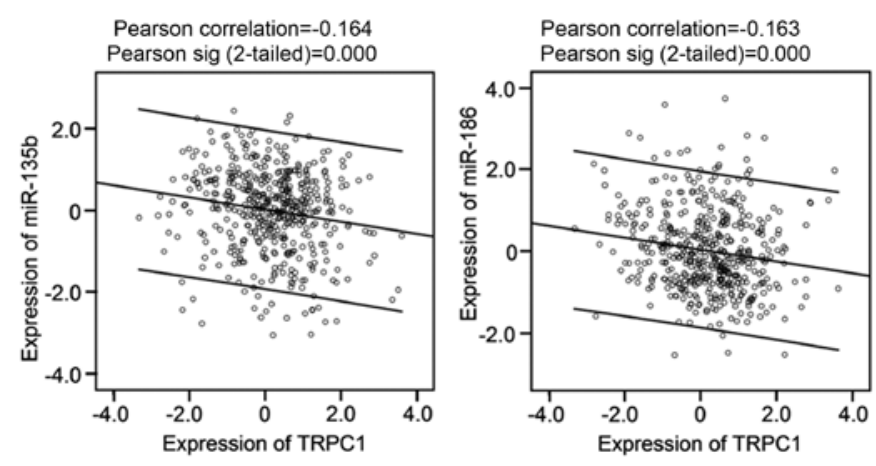

Figure 6. High expression of miR-135b and miR-186 are significantly correlated with the low expression of TRPC1 in ovarian cancer tissues. The expression data of microRNAs and TRPC1 in 489 cases of ovarian cancer tissues from a TCGA cohort was retrieved from cBioPortal for Cancer Genomics (http://cbioportal.org) $(23,24)$. The correlation between microRNAs and the gene was analyzed using Bivariate correlations.

Taxol resistance and is therefore of interest in the development of microRNA therapies for OC (69).

Taken together, these results provide further support for the involvement of TRPC1 in drug resistance in OC.

Low-level expression of TRPC1 correlates with higher histological tumor grade in a TCGA cohort. We next examined the expression profile and clinical significance of TRPC1 in a TCGA cohort. The clinical data of 489 patients with OC for whom TRPC1 mRNA expression data were available was retrieved from cBioPortal for Cancer Genomics (http://cbioportal.org) $(23,24)$. Data from the 341 patients with complete characteristics, including disease-free survival and status, overall survival and status, histological grade of the neoplasm, primary therapy outcome and tumor stage, were evaluated for an association of drug resistance in OC and TRPC1 expression. Gene expression was categorized high or low by the median value in accordance with a previous study (31). As shown in
Table III, TRPC1 expression differed significantly between patients with grade 2 (moderately-differentiated) $\mathrm{OC}$ and those with grade 3 (poorly-differentiated) OC ( $\mathrm{P}=0.006)$. Low-level expression correlated with higher tumor grade. A comparison of the disease-free and overall survival of the 341 OC patients with TRPC1 expression above or below the median level showed a disadvantage in terms of overall survival for patients with low vs. high expression $(48.700 \pm 2.307$ vs. $51.300 \pm 2.843)$, although the difference was not statistically significant.

\section{Discussion}

Tumorigenesis and tumor progression are driven by the aberrant function of genes that regulate genome stability, cell proliferation, apoptosis, angiogenesis, cell cycle, invasion, metastasis and drug resistance (89). With the rapid development of high-throughput technologies, databases such as GEO Profiles (22) and TCGA (90) have provided high-resolution molecular profiles of gene expression, microRNA expression, copy number and methylation in cancer tissues. In the present study, microarray data retrieved from GEO and TCGA were used to analyze TRPC1 expression in OC and its clinical relevance.

Bioinformatics analysis based on the various proteomic and genomic datasets can exploit the context of a protein/gene in cellular networks, provide insights into the functional role of a protein/gene, facilitate the rapid annotation of protein/gene function, and thereby guide laboratory experiments (91-94). For example, a comprehensive bioinformatics approach, including microarrays, motif analysis, protein/gene interaction, proteinchemical interaction, biological process annotation, pathway enrichment and mRNA-microRNA interactions, has been used to identify the association of dysregulated SPARCL1 and chemokine (C-C motif) ligand 21 (CCL21) (41), NIMArelated kinase 11 (NEK11) (95) and NIMA-related kinase 2 (NEK2) (96), and ribonuclease T2 (RNASET2) and gametogenetin binding protein 2 (GGNBP2) (97) with drug resistance in 
Table III. Correlation between TRPC1 expression and the clinicopathological characteristics of 341 patients with ovarian cancer.

\begin{tabular}{|c|c|c|c|c|}
\hline \multirow[b]{2}{*}{ Characteristics } & \multirow[b]{2}{*}{ No. of patients (\%) } & \multicolumn{2}{|c|}{ TRPC1 expression } & \multirow[b]{2}{*}{ P-value ${ }^{a}$} \\
\hline & & Low $(\%)$ & High $(\%)$ & \\
\hline Histological grade & & & & 0.006 \\
\hline Grade 2 & $43(12.6)$ & $13(30.2)$ & $30(69.8)$ & \\
\hline Grade 3 & $298(87.4)$ & $158(53.0)$ & $140(47.0)$ & \\
\hline Primary therapy outcome & & & & 0.352 \\
\hline Complete response & $261(76.5)$ & $136(52.1)$ & $125(47.9)$ & \\
\hline Partial response & 43 (12.6) & $18(41.9)$ & $25(58.1)$ & \\
\hline Progressive disease & $20(5.9)$ & $11(55.0)$ & $9(45.0)$ & \\
\hline Stable disease & $17(5.0)$ & $6(35.3)$ & $11(64.7)$ & \\
\hline Tumor stage & & & & 0.116 \\
\hline II & $20(5.9)$ & $13(65.0)$ & $7(35.0)$ & \\
\hline III & $270(79.2)$ & $138(51.1)$ & $132(48.9)$ & \\
\hline IV & $51(14.9)$ & $20(39.2)$ & $31(60.8)$ & \\
\hline
\end{tabular}

OC. In hepatocellular and gastric carcinomas, bioinformatics analysis revealed the association of E2F transcription factor 3 (E2F3) (98) and spalt-like transcription factor 4 (SALL4) (99), respectively, with prognosis. In this study, widely used bioinformatics tools and networks, including GeneMania (25), STITCH (version 4.0) (26,27), Coremine Medical (28), and DIANA miRPath (30), and six mRNA-microRNA prediction tools, including TargetScan, were used to analyze the associations of TRPC1 with drug resistance in OC.

TRPC1 mRNA expression was significantly and consistently downregulated by at least 3.260-fold in 251 OC samples compared with 24 normal control samples, according to three independent microarrays (Fig. 1A). In a previous study TRPC1 mRNA expression was lower in 5 ovarian serous papillary adenocarcinomas than in five normal samples (20). TRPC1 mRNA was at least 3-fold lower in OC cells than in normal ovarian surface epithelial cells (Fig. 1B). Significant downregulation of TRPC1 mRNA was also detected in cisplatin-resistant A2780 and SKOV3 cells and carboplatinresistant A2780 cells when compared with expression in their sensitive counterparts (Fig. 2). These results indicated that the stable and significant downregulation of TRPC1 in OC and drug-resistant cells plays a critical role in the development OC and in the regulation of drug resistance.

Further support for this conclusion was obtained in a comprehensive bioinformatics analyses. Protein/geneprotein/gene and protein-chemical interactions indicated the interaction of TRPC1 with 14 proteins/genes and 6 chemicals, all of which are associated with drug resistance in $\mathrm{OC}$ (Figs. 3 and 4). Annotation of TRPC1, OC and drug resistance supported a role for TRPC1 in drug-resistance-related functions in OC through 17 biological processes related to the cell cycle, gene expression and cell growth and cell death (Fig. 5). Among the top 11 pathways enriched from the top 38 microRNAs targeting TRPC1, 8 were involved in the regulation of drug resistance in OC (Table I). In addition, 8 of the top 10 microRNAs targeting TRPC1 were implicated in drug resistance in ovarian and other cancers (Table II).

A possible mechanism underlying the role of TRPC1 in drug resistance in $\mathrm{OC}$ is the regulation of autophagy. As shown in Fig. 3, co-expression and genetic interactions between TRPC1 and PIK3C3 and co-expression and co-localization with SPARCL1 were determined. PIK3C3 plays a critical role in the regulation of autophagy in vitro and in vivo (100). Autophagy, which acts both in protecting against cancer as well as promoting the growth of cancer, has attracted increased attention as an important mechanism for cancer development (101). Autophagy also contributes to drug resistance in ovarian and other cancers $(101,102)$. For instance, it has been reported that the induction of ERK-mediated autophagy conferred cisplatin resistance to ovarian cancer cells (103). The relationship between PIK3C3 and cancer has been targeted in chemotherapy via the drug paclitaxel, which has been used to block PIK3C3 activation (104). SPARCL1 was shown to be involved in the regulation of drug resistance via several pathways, including autophagy (41). The strong interactions of SPARCL1 with TRPC1 suggest the involvement of the latter in the regulation of autophagy and in drug resistance in $\mathrm{OC}$.

Finally, our analysis of the relationship between TRPC1 mRNA expression and the histological grade of OC in 341 patients of a TCGA cohort showed significant differences between grade 2 (moderately-differentiated) and grade 3 (poorly-differentiated) tumors, with the low-level expression of TRPC1 correlating with high tumor grade (Table III). This result provides clinical support for a link between the downregulation of TRPC1 and drug resistance in OC.

In summary, evidence obtained from RT-qPCR measurement, microarray data retrieval, comprehensive bioinformatics analyses, and clinical analysis of a TCGA cohort together suggest that the downregulation of TRPC1 contributes to drug resistance in OC and to the high histological grade of these 
tumors. Our results provide the basis for further investigation of the drug-resistance-related functions of TRPC1 in OC.

\section{Acknowledgements}

The present study was supported by the National Natural Science Foundation of China (grant nos. 81302283, 81560424 and 81460397), the China Postdoctoral Science Foundation (nos. 2014M552535XB and 2014M552291), the Natural Science Foundation of Guangxi (nos. 2014GXNSFCA118010, 2015GXNSFBA139115,2015GXNSFAA139151 and 2014GXNSFBA118155), and the Youth Science Foundation of Guangxi Medical University (no. GXMUYSF201312).

\section{References}

1. Matsuo K, Eno ML, Im DD, Rosenshein NB and Sood AK Clinical relevance of extent of extreme drug resistance in epithelial ovarian carcinoma. Gynecol Oncol 116: 61-65, 2010.

2. Cannistra SA: Cancer of the ovary. N Engl J Med 351: 2519-2529, 2004.

3. Agarwal R and Kaye SB: Ovarian cancer: Strategies for overcoming resistance to chemotherapy. Nat Rev Cancer 3: 502-516, 2003.

4. Parikh A, Lee C, Joseph P, Marchini S, Baccarini A, Kolev V, Romualdi C, Fruscio R, Shah H, Wang F, et al: microRNA-181a has a critical role in ovarian cancer progression through the regulation of the epithelial-mesenchymal transition. Nat Commun 5: 2977, 2014

5. Shah JS, Cole AJ, Dickson KA, Soon P and Marsh DJ: Investigating the role of long non-coding RNAs in cisplatin resistance in ovarian cancer. Asia Pac J Clin Oncol 10: 42-42, 2014.

6. Sorrentino A, Liu CG, Addario A, Peschle C, Scambia G and Ferlini C: Role of microRNAs in drug-resistant ovarian cancer cells. Gynecol Oncol 111: 478-486, 2008.

7. Suh DH, Kim MK, No JH, Chung HH and Song YS: Metabolic approaches to overcoming chemoresistance in ovarian cancer. Ann NY Acad Sci 1229: 53-60, 2011.

8. Yin F, Liu X, Li D, Wang Q, Zhang W and Li L: Tumor suppressor genes associated with drug resistance in ovarian cancer (Review). Oncol Rep 30: 3-10, 2013.

9. Richardson A and Kaye SB: Drug resistance in ovarian cancer: The emerging importance of gene transcription and spatio-temporal regulation of resistance. Drug Resist Updat 8: 311-321, 2005.

10. Liu X, Gao Y, Lu Y, Zhang J, Li L and Yin F: Oncogenes associated with drug resistance in ovarian cancer. J Cancer Res Clin Oncol 141: 381-395, 2015

11. Kahl CR and Means AR: Regulation of cell cycle progression by calcium/calmodulin-dependent pathways. Endocr Rev 24: 719-736, 2003

12. Roderick HL and Cook SJ: $\mathrm{Ca}^{2+}$ signalling checkpoints in cancer: Remodelling $\mathrm{Ca}^{2+}$ for cancer cell proliferation and survival. Nat Rev Cancer 8: 361-375, 2008.

13. Rajewskaya TA, Goncharova SA, Konovalova NP, Kotelnikova RA and Tatyanenko LV: Effect of drug resistance modulator, NO donor, on membrane structure and function of membrane-bound $\mathrm{Ca}^{2+}$-activated $\mathrm{Mg}^{2+}$-dependent ATPase. Bull Exp Biol Med 146: 200-202, 2008.

14. Clapham DE, Runnels LW and Strübing C: The TRP ion channel family. Nat Rev Neurosci 2: 387-396, 2001.

15. Nilius B and Szallasi A: Transient receptor potential channels as drug targets: From the science of basic research to the art of medicine. Pharmacol Rev 66: 676-814, 2014.

16. Ong HL and Ambudkar IS: The dynamic complexity of the TRPC1 channelosome. Channels (Austin) 5: 424-431, 2011.

17. Selli C, Erac Y and Tosun M: Simultaneous measurement of cytosolic and mitochondrial calcium levels: Observations in TRPC1-silenced hepatocellular carcinoma cells. J Pharmacol Toxicol Methods 72: 29-34, 2015.

18. He B, Liu F, Ruan J, Li A, Chen J, Li R, Shen J, Zheng D and Luo R: Silencing TRPC1 expression inhibits invasion of CNE2 nasopharyngeal tumor cells. Oncol Rep 27: 1548-1554, 2012.
19. Tajeddine $\mathrm{N}$ and Gailly P: TRPC1 protein channel is major regulator of epidermal growth factor receptor signaling. J Biol Chem 287: 16146-16157, 2012.

20. Zeng B, Yuan C, Yang X, Atkin SL and Xu SZ: TRPC channels and their splice variants are essential for promoting human ovarian cancer cell proliferation and tumorigenesis. Curr Cancer Drug Targets 13: 103-116, 2013.

21. Rhodes DR, Yu J, Shanker K, Deshpande N, Varambally R, Ghosh D, Barrette T, Pandey A and Chinnaiyan AM: ONCOMINE: A cancer microarray database and integrated data-mining platform. Neoplasia 6: 1-6, 2004.

22. Edgar R, Domrachev M and Lash AE: Gene Expression Omnibus: NCBI gene expression and hybridization array data repository. Nucleic Acids Res 30: 207-210, 2002.

23. Cerami E, Gao J, Dogrusoz U, Gross BE, Sumer SO, Aksoy BA, Jacobsen A, Byrne CJ, Heuer ML, Larsson E, et al: The cBio cancer genomics portal: An open platform for exploring multidimensional cancer genomics data. Cancer Discov 2: 401-404, 2012.

24. Gao J, Aksoy BA, Dogrusoz U, Dresdner G, Gross B, Sumer SO, Sun Y, Jacobsen A, Sinha R, Larsson E, et al: Integrative analysis of complex cancer genomics and clinical profiles using the cBioPortal. Sci Signal 6: pl1, 2013.

25. Zuberi K, Franz M, Rodriguez H, Montojo J, Lopes CT, Bader GD and Morris Q: GeneMANIA prediction server 2013 update. Nucleic Acids Res 41 (W1): W115-W122, 2013.

26. Kuhn M, von Mering C, Campillos M, Jensen LJ and Bork P: STITCH: Interaction networks of chemicals and proteins. Nucleic Acids Res 36 (Database): D684-D688, 2008.

27. Kuhn M, Szklarczyk D, Pletscher-Frankild S, Blicher TH, von Mering C, Jensen LJ and Bork P: STITCH 4: Integration of protein-chemical interactions with user data. Nucleic Acids Res 42 (D1): D401-D407, 2014.

28. de Leeuw N, Dijkhuizen T, Hehir-Kwa JY, Carter NP, Feuk L, Firth HV, Kuhn RM, Ledbetter DH, Martin CL, van RavenswaaijArts CM, et al: Diagnostic interpretation of array data using public databases and internet sources. Hum Mutat 33: 930-940, 2012.

29. Dweep H, Sticht C, Pandey P and Gretz N: miRWalk-database: Prediction of possible miRNA binding sites by 'walking' the genes of three genomes. J Biomed Inform 44: 839-847, 2011.

30. Vlachos IS, Kostoulas N, Vergoulis T, Georgakilas G, Reczko M, Maragkakis M, Paraskevopoulou MD, Prionidis K, Dalamagas T and Hatzigeorgiou AG: DIANA miRPath v.2.0: Investigating the combinatorial effect of microRNAs in pathways. Nucleic Acids Res 40 (W1): W498-W504, 2012.

31. Hedditch EL, Gao B, Russell AJ, Lu Y, Emmanuel C, Beesley J, Johnatty SE, Chen X, Harnett P, George J, et al; Australian Ovarian Cancer Study Group: ABCA transporter gene expression and poor outcome in epithelial ovarian cancer. J Natl Cancer Inst 106: 106, 2014.

32. Meng D, Chen Y, Zhao Y, Wang J, Yun D, Yang S, Chen J, Chen H and Lu D: Expression and prognostic significance of TCTN1 in human glioblastoma. J Transl Med 12: 288, 2014.

33. Bowen NJ, Walker LD, Matyunina LV, Logani S, Totten KA, Benigno BB and McDonald JF: Gene expression profiling supports the hypothesis that human ovarian surface epithelia are multipotent and capable of serving as ovarian cancer initiating cells. BMC Med Genomics 2: 71, 2009.

34. Li M, Balch C, Montgomery JS, Jeong M, Chung JH, Yan P, Huang TH, Kim S and Nephew KP: Integrated analysis of DNA methylation and gene expression reveals specific signaling pathways associated with platinum resistance in ovarian cancer. BMC Med Genomics 2: 34, 2009.

35. Peters D, Freund J and Ochs RL: Genome-wide transcriptional analysis of carboplatin response in chemosensitive and chemoresistant ovarian cancer cells. Mol Cancer Ther 4: 1605-1616, 2005.

36. Lee S, Choi EJ, Jin C and Kim DH: Activation of PI3K/Akt pathway by PTEN reduction and PIK3CA mRNA amplification contributes to cisplatin resistance in an ovarian cancer cell line. Gynecol Oncol 97: 26-34, 2005.

37. Wu H, Cao Y, Weng D, Xing H, Song X, Zhou J, Xu G, Lu Y, Wang S and Ma D: Effect of tumor suppressor gene PTEN on the resistance to cisplatin in human ovarian cancer cell lines and related mechanisms. Cancer Lett 271: 260-271, 2008.

38. Yan X, Fraser M, Qiu Q and Tsang BK: Over-expression of PTEN sensitizes human ovarian cancer cells to cisplatin-induced apoptosis in a p53-dependent manner. Gynecol Oncol 102: 348-355, 2006. 
39. Wu H, Wang K, Liu W and Hao Q: PTEN overexpression improves cisplatin-resistance of human ovarian cancer cells through upregulating KRT10 expression. Biochem Biophys Res Commun 444: 141-146, 2014

40. Zhang X, Wang X, Song X, Liu C, Shi Y, Wang Y, Afonja O, $\mathrm{Ma}$ C, Chen YH and Zhang L: Programmed cell death 4 enhances chemosensitivity of ovarian cancer cells by activating death receptor pathway in vitro and in vivo. Cancer Sci 101: 2163-2170, 2010.

41. Yin F, Liu X, Li D, Wang Q, Zhang W and Li L: Bioinformatic analysis of chemokine (C-C motif) ligand 21 and SPARC-like protein 1 revealing their associations with drug resistance in ovarian cancer. Int J Oncol 42: 1305-1316, 2013.

42. Sen T, Sen N, Noordhuis MG, Ravi R, Wu TC,Ha PK, Sidransky D and Hoque MO: OGDHL is a modifier of AKT-dependent signaling and NF-кB function. PLoS One 7: e48770, 2012.

43. Xia X, Ma Q, Li X, Ji T, Chen P, Xu H, Li K, Fang Y, Weng D, Weng Y, et al: Cytoplasmic p21 is a potential predictor for cisplatin sensitivity in ovarian cancer. BMC Cancer 11: 399, 2011.

44. Materna V, Surowiak P, Markwitz E, Spaczynski M, DragZalesinska $\mathrm{M}$, Zabel $\mathrm{M}$ and Lage $\mathrm{H}$ : Expression of factors involved in regulation of DNA mismatch repair- and apoptosis pathways in ovarian cancer patients. Oncol Rep 17: 505-516, 2007.

45. Moorehead RA and Singh G: Influence of the proto-oncogene c-fos on cisplatin sensitivity. Biochem Pharmacol 59: 337-345, 2000.

46. Mahner S, Baasch C, Schwarz J, Hein S, Wölber L, Jänicke F and Milde-Langosch $\mathrm{K}$ : C-Fos expression is a molecular predictor of progression and survival in epithelial ovarian carcinoma. Br J Cancer 99: 1269-1275, 2008.

47. Liu YY, Li L, Li DR, Zhang W and Wang Q: Suppression of WWOX gene by RNA interference reverses platinum resistance acquired in SKOV3/SB cells. Zhonghua Fu Chan Ke Za Zhi 43 : 854-858, 2008 (In Chinese).

48. Liu T, Zhao L, Chen W, Li Z, Hou H, Ding L and Li X: Inactivation of von Hippel-Lindau increases ovarian cancer cell aggressiveness through the HIF1 $\alpha / \mathrm{miR}-210 / \mathrm{VMP} 1$ signaling pathway. Int J Mol Med 33: 1236-1242, 2014.

49. Hamada $H$, Hagiwara $K$, Nakajima $T$ and Tsuruo $T$ : Phosphorylation of the $\mathrm{Mr} 170,000$ to 180,000 glycoprotein specific to multidrug-resistant tumor cells: Effects of verapamil, trifluoperazine, and phorbol esters. Cancer Res 47: 2860-2865, 1987.

50. Zhao BX, Sun YB, Wang SQ, Duan L, Huo QL, Ren F and Li GF: Grape seed procyanidin reversal of P-glycoprotein associated multi-drug resistance via down-regulation of NF- $\kappa \mathrm{B}$ and MAPK/ERK mediated YB-1 activity in A2780/T cells. PLoS One 8: e71071, 2013.

51. Lee LF, Haskill JS, Mukaida N, Matsushima K and Ting JP: Identification of tumor-specific paclitaxel (Taxol)-responsive regulatory elements in the interleukin-8 promoter. Mol Cell Biol 17: 5097-5105, 1997

52. Duan Z, Feller AJ, Penson RT, Chabner BA and Seiden MV: Discovery of differentially expressed genes associated with paclitaxel resistance using cDNA array technology: Analysis of interleukin (IL) 6, IL-8, and monocyte chemotactic protein 1 in the paclitaxel-resistant phenotype. Clin Cancer Res 5: 3445-3453, 1999.

53. Huang Y, Ju B, Tian J, Liu F, Yu H, Xiao H, Liu X, Liu W, Yao Z and Hao Q: Ovarian cancer stem cell-specific gene expression profiling and targeted drug prescreening. Oncol Rep 31: 1235-1248, 2014

54. Engelmann BJ, Ryan JJ and Farrell NP: Antidepressants and platinum drugs. Anticancer Res 34: 509-516, 2014.

55. Lee CS, Kim YJ, Jang ER, Kim W and Myung SC: Fluoxetine induces apoptosis in ovarian carcinoma cell line OVCAR-3 through reactive oxygen species-dependent activation of nuclear factor-kappaB. Basic Clin Pharmacol Toxicol 106: 446-453, 2010.

56. Hiss DC, Gabriels GA and Folb PI: Combination of tunicamycin with anticancer drugs synergistically enhances their toxicity in multidrug-resistant human ovarian cystadenocarcinoma cells. Cancer Cell Int 7: 5, 2007.

57. Rogan AM, Hamilton TC, Young RC, Klecker RW Jr and Ozols RF: Reversal of adriamycin resistance by verapamil in human ovarian cancer. Science 224: 994-996, 1984.
58. Ozols RF: Pharmacologic reversal of drug resistance in ovarian cancer. Semin Oncol 12 (Suppl 4): 7-11, 1985

59. Gene Ontology consortium: http://www.geneontology.org.

60. Gamberoni G, Storari S and Volinia S: Finding biological process modifications in cancer tissues by mining gene expression correlations. BMC Bioinformatics 7: 6, 2006.

61. Lagreid A, Hvidsten TR, Midelfart H, Komorowski J and Sandvik AK: Predicting gene ontology biological process from temporal gene expression patterns. Genome Res 13: 965-979, 2003.

62. Medical COREMINE: http://www.coremine.com/medical/.

63. Kloosterman WP and Plasterk RH: The diverse functions of microRNAs in animal development and disease. Dev Cell 11: 441-450, 2006

64. Croce CM and Calin GA: miRNAs, cancer, and stem cell division. Cell 122: 6-7, 2005.

65. Tili E, Michaille JJ, Gandhi V, Plunkett W, Sampath D and Calin GA: miRNAs and their potential for use against cancer and other diseases. Future Oncol 3: 521-537, 2007.

66. Brazil DP, Park J and Hemmings BA: PKB binding proteins. Getting in on the Akt. Cell 111: 293-303, 2002.

67. Tang W, Jiang Y, Mu X, Xu L, Cheng W and Wang X: MiR-135a functions as a tumor suppressor in epithelial ovarian cancer and regulates HOXA10 expression. Cell Signal 26: 1420-1426, 2014.

68. Matei D, Fang F, Shen C, Schilder J, Arnold A, Zeng Y, Berry WA, Huang T and Nephew KP: Epigenetic resensitization to platinum in ovarian cancer. Cancer Res 72: 2197-2205, 2012.

69. Kim YW, Kim EY, Jeon D, Liu JL, Kim HS, Choi JW and Ahn WS: Differential microRNA expression signatures and cell type-specific association with Taxol resistance in ovarian cancer cells. Drug Des Devel Ther 8: 293-314, 2014.

70. Arafa SA, Zhu Q, Barakat BM, Wani G, Zhao Q, El-Mahdy MA and Wani AA: Tangeretin sensitizes cisplatin-resistant human ovarian cancer cells through downregulation of phosphoinositide 3-kinase/Akt signaling pathway. Cancer Res 69: 8910-8917, 2009.

71. Lange TS, Stuckey AR, Robison K, Kim KK, Singh RK, Raker CA and Brard L: Effect of a vitamin $\mathrm{D}_{3}$ derivative (B3CD) with postulated anti-cancer activity in an ovarian cancer animal model. Invest New Drugs 28: 543-553, 2010.

72. Jiao JW and Wen F: Tanshinone IIA acts via p38 MAPK to induce apoptosis and the down-regulation of ERCC1 and lung-resistance protein in cisplatin-resistant ovarian cancer cells. Oncol Rep 25: 781-788, 2011.

73. Kumar S, Kumar A, Shah PP, Rai SN, Panguluri SK and Kakar SS: MicroRNA signature of cis-platin resistant vs. cisplatin sensitive ovarian cancer cell lines. J Ovarian Res 4: 17, 2011.

74. Jin L, Huo Y, Zheng Z, Jiang X, Deng H, Chen Y, Lian Q, Ge $\mathrm{R}$ and Deng H: Down-regulation of Ras-related protein Rab 5C-dependent endocytosis and glycolysis in cisplatin-resistant ovarian cancer cell lines. Mol Cell Proteomics 13: 3138-3151, 2014.

75. Li J, Zhang Y, Gao Y, Cui Y, Liu H, Li M and Tian Y: Downregulation of HNF1 homeobox B is associated with drug resistance in ovarian cancer. Oncol Rep 32: 979-988, 2014.

76. Rosanò L, Cianfrocca R, Tocci P, Spinella F, Di Castro V, Caprara V, Semprucci E, Ferrandina G, Natali PG and Bagnato A: Endothelin A receptor/ $\beta$-arrestin signaling to the Wnt pathway renders ovarian cancer cells resistant to chemotherapy. Cancer Res 74: 7453-7464, 2014.

77. Ko MA, Zehong G, Virtanen C, Guindi M, Waddell TK, Keshavjee S, et al: MicroRNA expression profiling of esophageal cancer before and after induction chemoradiotherapy. Ann Thorac Surg 94: 1094-1102; discussion 1102-1093, 2012.

78. Wang FJ, Ding Y, Mao YY, Jing FY, Zhang ZY, Jiang LF, Guo JF, Sun XJ, Jin MJ and Chen K: Associations between hsa-miR-603 polymorphism, lifestyle-related factors and colorectal cancer risk. Cancer Biomark 14: 225-231, 2014.

79. Rogler A, Hoja S, Socher E, Nolte E, Wach S, Wieland W, Hofstädter F, Goebell PJ, Wullich B, Hartmann A, et al: Role of two single nucleotide polymorphisms in secreted frizzled related protein 1 and bladder cancer risk. Int J Clin Exp Pathol 6: 1984-1998, 2013

80. Zhang J, Zhang T, Ti X, Shi J, Wu C, Ren X and Yin $\mathrm{H}$ Curcumin promotes apoptosis in A549/DDP multidrug-resistant human lung adenocarcinoma cells through an miRNA signaling pathway. Biochem Biophys Res Commun 399: 1-6, 2010. 
81. Zhu W, Zhu D, Lu S, Wang T, Wang J, Jiang B, Shu Y and Liu P: miR-497 modulates multidrug resistance of human cancer cell lines by targeting BCL2. Med Oncol 29: 384-391, 2012.

82. Della Vittoria Scarpati G, Falcetta F, Carlomagno C, Ubezio P, Marchini S, De Stefano A, Singh VK, D'Incalci M, De Placido S and Pepe S: A specific miRNA signature correlates with complete pathological response to neoadjuvant chemoradiotherapy in locally advanced rectal cancer. Int J Radiat Oncol Biol Phys 83 1113-1119, 2012

83. Tang J, Tao ZH, Wen D, Wan JL, Liu DL, Zhang S, Cui JF, Sun HC, Wang L, Zhou J, et al: MiR-612 suppresses the stemness of liver cancer via Wnt/ $\beta$-catenin signaling. Biochem Biophys Res Commun 447: 210-215, 2014.

84. Yang Y, Li H, Hou S, Hu B, Liu J and Wang J: The noncoding RNA expression profile and the effect of lncRNA AK126698 on cisplatin resistance in non-small-cell lung cancer cell. PLoS One 8: e65309, 2013

85. Ichikawa T, Sato F, Terasawa K, Tsuchiya S, Toi M, Tsujimoto G and Shimizu K: Trastuzumab produces therapeutic actions by upregulating miR-26a and miR-30b in breast cancer cells. PLoS One 7: e31422, 2012.

86. Gu YF, Zhang H, Su D, Mo ML, Song P, Zhang F and Zhang SC: miR-30b and miR-30c expression predicted response to tyrosine kinase inhibitors as first line treatment in non-small cell lung cancer. Chin Med J (Engl) 126: 4435-4439, 2013.

87. Pichiorri F, Palmieri D, De Luca L, Consiglio J, You J, Rocci A, Talabere T, Piovan C, Lagana A, Cascione L, et al: In vivo NCL targeting affects breast cancer aggressiveness through miRNA regulation. J Exp Med 210: 951-968, 2013.

88. Huang JW, Wang Y, Dhillon KK, Calses P, Villegas E, Mitchell PS, Tewari M, Kemp CJ and Taniguchi T: Systematic screen identifies miRNAs that target RAD51 and RAD51D to enhance chemosensitivity. Mol Cancer Res 11: 1564-1573, 2013.

89. Huang N, Shah PK and Li C: Lessons from a decade of integrating cancer copy number alterations with gene expression profiles. Brief Bioinform 13: 305-316, 2012.

90. McLendon R, Friedman A, Bigner D, Van Meir EG, Brat DJ, Mastrogianakis GM, Olson JJ, Mikkelsen T, Lehman N, Aldape K, et al; Cancer Genome Atlas Research Network: Comprehensive genomic characterization defines human glioblastoma genes and core pathways. Nature 455: 1061-1068, 2008.

91. Sharan R, Ulitsky I and Shamir R: Network-based prediction of protein function. Mol Syst Biol 3: 88, 2007.
92. Phuong T and Nhung N: Predicting gene function using similarity learning. BMC Genomics 14 (Suppl 4): S4, 2013.

93. Janga SC, Díaz-Mejía JJ and Moreno-Hagelsieb G: Networkbased function prediction and interactomics: The case for metabolic enzymes. Metab Eng 13: 1-10, 2011.

94. Yu G, Zhu H, Domeniconi C and Guo M: Integrating multiple networks for protein function prediction. BMC Syst Biol 9 (Suppl 1): S3, 2015

95. Liu X, Gao Y, Lu Y, Zhang J, Li L and Yin F: Downregulation of NEK11 is associated with drug resistance in ovarian cancer. Int J Oncol 45: 1266-1274, 2014

96. Liu X, Gao Y, Lu Y, Zhang J, Li L and Yin F: Upregulation of NEK2 is associated with drug resistance in ovarian cancer. Oncol Rep 31: 745-754, 2014.

97. Yin F, Liu L, Liu X, Li G, Zheng L, Li D, Wang Q, Zhang W and Li L: Downregulation of tumor suppressor gene ribonuclease $\mathrm{T} 2$ and gametogenetin binding protein 2 is associated with drug resistance in ovarian cancer. Oncol Rep 32: 362-372, 2014.

98.Zeng X, Yin F, Liu X, Xu J, Xu Y, Huang J, Nan Y and Qiu X: Upregulation of E2F transcription factor 3 is associated with poor prognosis in hepatocellular carcinoma. Oncol Rep 31: 1139-1146, 2014.

99. Liu J, Wang LY, Yang AJ, Jiang PF and Wang MC: Up-regulation of SALL4 associated with poor prognosis in gastric cancer. Hepatogastroenterology 61: 1459-1464, 2014

100. Jaber N, Dou Z, Lin RZ, Zhang J and Zong WX: Mammalian PIK3C3/VPS34: The key to autophagic processing in liver and heart. Autophagy 8: 707-708, 2012.

101. Yang ZJ, Chee CE, Huang S and Sinicrope FA: The role of autophagy in cancer: Therapeutic implications. Mol Cancer Ther 10: 1533-1541, 2011

102. Peracchio C, Alabiso O, Valente $G$ and Isidoro $C$ : Involvement of autophagy in ovarian cancer: A working hypothesis. J Ovarian Res 5: 22, 2012.

103. Wang J and Wu GS: Role of autophagy in cisplatin resistance in ovarian cancer cells. J Biol Chem 289: 17163-17173, 2014.

104. Veldhoen RA, Banman SL, Hemmerling DR, Odsen R, Simmen T, Simmonds AJ, Underhill DA and Goping IS: The chemotherapeutic agent paclitaxel inhibits autophagy through two distinct mechanisms that regulate apoptosis. Oncogene 32 : 736-746, 2013. 